\title{
Pore Space Connectivity and the Transport Properties of Rocks
}

\author{
Yves Bernabé ${ }^{{ }^{*}}$, Min Li ${ }^{2}$, Yan-Bing Tang ${ }^{2}$ and Brian Evans ${ }^{1}$ \\ ${ }^{1}$ Earth, Atmospheric and Planetary Sciences Department, Massachusetts Institute of Technology, Cambridge, Massachusetts - USA \\ 2 State Key Laboratory of Oil and Gas Reservoir Geology and Exploitation, Southwest Petroleum University, Chengdu - China \\ e-mail: yvb@mit.edu \\ * Corresponding author
}

\begin{abstract}
Pore connectivity is likely one of the most important factors affecting the permeability of reservoir rocks. Furthermore, connectivity effects are not restricted to materials approaching a percolation transition but can continuously and gradually occur in rocks undergoing geological processes such as mechanical and chemical diagenesis. In this study, we compiled sets of published measurements of porosity, permeability and formation factor, performed in samples of unconsolidated granular aggregates, in which connectivity does not change, and in two other materials, sintered glass beads and Fontainebleau sandstone, in which connectivity does change. We compared these data to the predictions of a Kozeny-Carman model of permeability, which does not account for variations in connectivity, and to those of Bernabé et al. $(2010,2011)$ model, which does [Bernabé Y., Li M., Maineult A. (2010) Permeability and pore connectivity: a new model based on network simulations, J. Geophys. Res. 115, B10203; Bernabé Y., Zamora M., Li M., Maineult A., Tang Y.B. (2011) Pore connectivity, permeability and electrical formation factor: a new model and comparison to experimental data, J. Geophys. Res. 116, B11204]. Both models agreed equally well with experimental data obtained in unconsolidated granular media. But, in the other materials, especially in the low porosity samples that had undergone the greatest amount of sintering or diagenesis, only Bernabé et al. model matched the experimental data satisfactorily. In comparison, predictions of the Kozeny-Carman model differed by orders of magnitude. The advantage of the Bernabé et al. model was its ability to account for a continuous, gradual reduction in pore connectivity during sintering or diagenesis. Although we can only speculate at this juncture about the mechanisms responsible for the connectivity reduction, we propose two possible mechanisms, likely to be active at different stages of sintering and diagenesis, and thus allowing the gradual evolution observed experimentally.
\end{abstract}

Résumé - Connectivité de l'espace poreux et propriétés de transport des roches — La connectivité porale est probablement l'un des plus importants facteurs affectant la perméabilité des roches réservoir. En outre, les effets de connectivité ne sont pas limités aux seuls matériaux approchant un seuil de percolation mais peuvent avoir lieu progressivement dans des roches soumises à des processus tels que la diagénèse mécanique ou chimique. Dans cette étude, nous avons compilé plusieurs jeux, préalablement publiés, de mesures de porosité, perméabilité et facteur de formation, obtenues sur des agrégats granulaires non-consolidés, pour lesquels la connectivité ne change pas, ainsi que dans deux autres matériaux, billes de verre frittées et grès de Fontainebleau, pour lesquels elle varie. Nous avons comparé ces données aux prédictions d'un modèle de perméabilité de type Kozeny-Carman, qui ne 
tient pas compte des variations de connectivité, et à celles du modèle de Bernabé et al. (2010, 2011), qui les inclut [Bernabé Y., Li M., Maineult A. (2010) Permeability and pore connectivity: a new model based on network simulations, J. Geophys. Res. 115, B10203; Bernabé Y., Zamora M., Li M., Maineult A., Tang Y.B. (2011) Pore connectivity, permeability and electrical formation factor: a new model and comparison to experimental data, J. Geophys. Res. 116, B11204]. Les deux modèles sont apparus également en bon accord avec les données expérimentales pour les agrégats granulaires nonconsolidés. En revanche, pour les deux autres matériaux, en particulier dans les échantillons les moins poreux, qui ont été le plus longtemps soumis au frittage ou à la diagénèse, les données expérimentales n'ont pu être expliquées que par le seul modèle de Bernabé et al. En comparaison, les prédictions du modèle de type Kozeny-Carman étaient erronées de plusieurs ordres de grandeur. La valeur du modèle de Bernabé et al. réside dans sa capacité à reproduire la réduction continue et progressive de la connectivité ayant lieu lors du frittage ou de la diagénèse. Bien que nous ne puissions, à ce stade, que spéculer sur les mécanismes responsables de la réduction de connectivité, nous proposons deux mécanismes possibles, susceptibles d'être actifs à des étapes différentes du frittage et de la diagénèse, et donc de permettre l'évolution continue observée expérimentalement.

\section{INTRODUCTION}

Permeability is the most important rock property controlling oil and gas production rates. There is great interest in the oil and gas industry for devising reliable and accurate models of permeability that could be applied to core, well-log and geophysical data. Dimensional analysis shows that permeability is the product of a length scale squared by a dimensionless, scale-invariant quantity (Berryman, 1992ab, 1993; Bernabé et al., 2010), where "scale-invariant" refers to invariance under application to the porous medium of a transform such that the distance between any pair of points in the medium is scaled by a constant factor. The choice of a length-scale parameter is arbitrary (Bernabé et al., 2010). The only qualities required of an effective length-scale parameter are that it should be unambiguously defined, and that it should be measurable in rock samples, preferably through a variety of independent techniques. One suitable length-scale is the hydraulic radius $r_{H}$, here defined as twice the volume to surface ratio of the pore space $\left(r_{H}=2 V_{p} / A_{p}\right.$, where $V_{p}$ and $A_{p}$ are the pore volume and internal wetted surface area, respectively). The hydraulic radius can be measured by several methods, including picnometry, the BET gas-adsorption technique, or microstructural image analysis. The form of the scale-invariant term is more difficult to determine. Fluid flow through porous media is affected to various degrees by a vast number of scale-invariant factors (e.g., normalized moments of the pore size and pore-length distributions, pore aspect ratio, relative pore-wall roughness, pore connectivity, and so forth). Therefore, identifying the major factors and their functional form in a permeability model is a challenging task.

Our main goals here are to show that pore connectivity is one of the most important scale-invariant factors and, furthermore, that connectivity effects are not limited to media going through a percolation transition but can gradually occur in rocks undergoing common geological processes such as mechanical and chemical diagenesis, microfracturing during uplift, and so forth. These continuous changes in connectivity can alter permeability in ways that are not predicted using widely held models such as the equivalent channel model of Paterson (1983) and Walsh and Brace (1984) (PWB), which do not explicitly include pore connectivity. Our approach will be to analyze experimental datasets in porous materials subject to processes expected to affect differently the length scale $r_{H}$ and the pore connectivity. As a result, these materials display very different interrelationships among porosity $(\phi)$, permeability $(k)$ and formation factor $(F)$, which can then be used to identify and quantify the effect of the connectivity variations. In particular, we will show that the experimental data can be better explained using the connectivity models such as Bernabé et al. $(2010,2011)$.

The paper is organized as follows. In Section 1, we briefly present the permeability and formation factor models used to interpret the experimental data. The materials selected for this study and the associated datasets are described in Section 2. The data are analyzed in Section 3 using the models of Section 1. The results are discussed in Section 4 and our conclusions presented at the end.

\section{PERMEABILITY AND FORMATION FACTOR MODELS}

The PWB model belongs to the Kozeny-Carman class of models. According to PWB, the pore space can be represented by a single characteristic channel having the appropriate dimensions, shape and orientation. 
When simple channel shapes such as circular cylinders or flat slits are considered, it is easy to derive the following equations:

$$
\begin{gathered}
k=\frac{D \phi r_{H}^{2}}{\tau^{2}} \\
F=\frac{\tau^{2}}{\phi}
\end{gathered}
$$

and

$$
k=\frac{D r_{H}^{2}}{F}
$$

where $D$ is a geometric form factor equal to $1 / 8$ for a circular cylinder and $1 / 12$ for a flat slit, and $\tau$ denotes the tortuosity, i.e., the ratio of the channel length to the nominal length of the corresponding porous medium. Inspection of Equations (1-3) shows that the PWB model includes three scale-invariant parameters, $D, \phi$ and $\tau$, corresponding to the shape, volume fraction and orientation of the pores. If a strict geometrical definition is used for $D$ and $\tau$, they should both be expected to display a very limited range of variation (i.e., between $1 / 8$ to $1 / 12$ for $D$ and around square root of 2 for $\tau$ ). These ranges have often been found inadequate, and tortuosity, in particular, is frequently assumed to account implicitly for a host of effects not directly included in PWB (Clennell, 1997, for a thorough review of tortuosity). However, this practice transforms tortuosity into an ill-defined parameter, which cannot be measured independently. Equation (2) can be replaced or supplemented by Archie's law $F=\phi^{-m}$ (Revil and Cathles, 1999; Glover et al., 2006), in which the cementation exponent $m$ is interpreted as an indicator of the pore space "connectedness" (Glover, 2009).

Bernabé et al. $(2010,2011)$ recently proposed an alternative model, in which connectivity is explicitly included. The model assumes that pore-scale heterogeneity and connectivity are the major scale-invariant factors controlling $k$ and $F$. For the sake of simplicity, pore-scale heterogeneity is restricted to cross-sectional pore size variability and is measured by the standard deviation $(\sigma)$ of the pore radius distribution normalized to the mean radius. Additional heterogeneity parameters may be needed for rocks with very complex pore shapes (e.g., carbonates) and/or a bimodal pore size distribution (e.g., presence of a micro-porous phase). Bernabé et al. (2010, 2011) notion of connectivity is similar to Glover's "connectedness", i.e., "general availability of pathways for transport" (Glover, 2009). According to this definition, a natural measure of pore connectivity is the mean coordination number $(z)$, i.e., the average number of channels connected to a nodal pore (Bernabé and Maineult, 2015, for a discussion of $z$ as pore connectivity measure). Bernabé et al. model is summarized in Equations (4-6):

$$
\begin{aligned}
& k=C_{k}(\sigma, \varepsilon)\left(\frac{r_{\mathrm{H}}}{l}\right)^{2}\left(z-z_{c}\right)^{\beta(\sigma)} r_{\mathrm{H}}^{2} \\
& 1 / F=C_{F}(\sigma, \varepsilon)\left(\frac{r_{\mathrm{H}}}{l}\right)^{2}\left(z-z_{c}\right)^{\gamma(\sigma)}
\end{aligned}
$$

and

$$
k=C(\sigma, \varepsilon)\left(\frac{r_{\mathrm{H}}}{l}\right)^{2(1-\alpha(\sigma))}(1 / F)^{\alpha(\sigma)} r_{\mathrm{H}}^{2}
$$

where the exponents $(\beta, \gamma, \alpha=\beta / \gamma)$ are functions of the porescale heterogeneity measure $\sigma$ while the pre-factors $\left(C_{k}, C_{F}\right.$, $C=C_{k} C_{F}^{-\alpha}$ ) depend on $\sigma$ and $\varepsilon$, where $\varepsilon$ refers to the mean aspect ratio of the approximately elliptical pore crosssections. Approximate functional forms for the pre-factors and exponents were determined from the results of network simulations (Bernabé et al., 2010, 2011). They are repeated here for convenience in Table 1 . The four scale-invariant parameters used in the model, i.e., $\sigma, z, \varepsilon$ and the normalized pore length $l / r_{H}$, can, in principle, all be measured in two- and three-dimensional images of the pore space of rock samples.

Before moving on to the rest of the study, we note that the classic definition of the formation factor, i.e., the ratio of the sample resistivity to that of the saturating brine is formally incorrect because an electrical double layer usually forms at the mineral/water interface, giving rise to anomalous surface conduction that may significantly affect the rock conductivity (Revil and Glover, 1997, 1998). Neglecting surface conduction leads to an underestimation of the formation factor that is approximately:

$$
F^{*} \approx F\left(1-2 \Sigma_{S} / r_{H} \sigma_{b r}\right)
$$

where $F^{*}$ is the apparent formation factor, $\Sigma_{S}$ the specific surface conductivity and $\sigma_{b r}$ the brine conductivity. In clay-free sandstones saturated with a high-salinity aqueous solution the ratio $\Sigma_{S} / r_{H} \sigma_{b r}$ is much smaller than 1 and surface conduction can be neglected. This condition is assumed to hold in the rest of the paper.

\section{MATERIALS: TRANSPORT PROPERTIES AND HYDRAULIC RADIUS}

\subsection{Unconsolidated Glass Beads and Sand}

If properly prepared, random packs of monodisperse spherical grains should have statistically equivalent structures with identical scale-invariant parameters, independent of the radius of the spheres. The PWB and Bernabé et al. models both predict that, in such sphere packs, permeability 
TABLE 1

Approximate expressions of the exponents and pre-factors of Equations (4-6) as functions of $\sigma$ and $\varepsilon$ (from Bernabé et al., 2011)

\begin{tabular}{c|c}
\hline$\beta=1.2343+0.93462 \sigma+1.4755 \sigma^{2}$ & $C_{k}=\psi_{k}(\varepsilon) 10^{-\left(1.1950+0.82190 \sigma+2.0459 \sigma^{2}\right)}, \psi_{k}(\varepsilon)=\frac{\left[3(1+\varepsilon)-\sqrt{3 \varepsilon^{2}+10 \varepsilon+3}\right]^{2}}{8 \varepsilon\left(1+\varepsilon^{2}\right)}$ \\
\hline$\gamma=1.2903+0.045527 \sigma+0.82390 \sigma^{2}$ & $C_{F}=\psi_{F}(\varepsilon) 10^{-\left(0.32894+0.23339 \sigma+1.1423 \sigma^{2}\right)}, \psi_{F}(\varepsilon)=\frac{\left[3(1+\varepsilon)-\sqrt{3 \varepsilon^{2}+10 \varepsilon+3}\right]^{2}}{4 \varepsilon}$ \\
\hline$\alpha=\frac{\beta}{\gamma}$ & $C=C_{k} C_{F}^{-\alpha}$ \\
\hline
\end{tabular}

scales as $r_{H}^{2}$ while the formation factor remains constant. The same should be approximately true of unconsolidated packs of well-sorted, slightly non-spherical particles. To test this we considered the experimental data collected on unconsolidated packs of monodisperse spherical glass beads (Glover and Walker, 2009; Glover and Déry, 2010) and well-sorted, rounded sands (Biella and Tabacco, 1981; Biella et al., 1983). These datasets include values of porosity, grain radius, permeability and formation factor (note that $F$ was measured in the high salinity limit, with negligible surface conduction). As can be seen in Figure 1, the porosity $\phi$ and formation factor $F$ of unconsolidated, well-sorted granular media are nearly constant, equal to 0.39 and 4.3 , respectively, while the permeability $k$ varies over 6 orders of magnitude from about 0.1 to $10000.10^{-12} \mathrm{~m}^{2}$ (or Darcy). These values of $\phi$ and $F$ correspond to a cementation exponent of 1.5 in good accordance with theoretical predictions (Glover et al., 2000). A minor trend is visible in the data with high permeabilities corresponding to low porosities and high formation factors, and vice versa (Fig. 1a, c). This effect is more pronounced in sands than in glass beads and may reflect the existence of a weak correlation of grain size to grain asphericity (Bernabé et al., 2011) and/or packing structure. In the papers cited above, the experimental errors were on the order of few percent, i.e., substantially lower than the sample-to-sample fluctuations occurring during the preparation of the bead and sand packs. Sample-to-sample variability was even more prevalent in sintered glass beads and Fontainebleau sandstone as shown in Figure 1.

The hydraulic radius of a pack of perfectly monodisperse spheres is given by:

$$
r_{H}=\frac{2 \phi R_{G}}{3(1-\phi)}
$$

where $R_{G}$ denotes the sphere radius (see the derivation in Appendix A). Note that Equation (8) considers a sphere pack of infinite extent and does not include the end effect associated with the walls of the container that must always be used in practice. Obviously, this end effect is negligible when the grain size is significantly lower than the container size, a condition that appears to be met in the studies mentioned above, except possibly for the millimeter-size glass beads of Glover and Walker (2009), who used relatively small containers $(2.5 \mathrm{~cm}$ diameter $)$. Based on the small experimental errors reported for $\phi$ and $R_{G}$, the relative uncertainty $\delta r_{H} / r_{H}$ was estimated to be lower than $\pm 5 \%$ (Fig. 2).

In the case of sand packs, the sand grains are not spherical and Equation (8) must be modified as:

$$
r_{H}=\frac{2 \phi R_{G}}{3 f_{G}(1-\phi)}
$$

where the grain radius $R_{G}$ is now defined as the radius of the sphere with an equal volume $V_{G}=4 \pi R_{G}{ }^{3} / 3$ and the asphericity factor $f_{G}>1$ is equal to the ratio of the grain surface area $A_{G}$ to that of the equivalent sphere, $f_{G}=A_{G} / 4 \pi R_{G}{ }^{2}$. In order to estimate $f_{G}$, we applied two previously proposed formulas linking $f_{G}$ and $\phi$ for packs of ellipsoidal grains (Coelho et al., 1997) and found $f_{G}$ values increasing from 1 for the coarsest sands to about 1.3 for the finest ones (this increase of ellipticity with decreasing grain size was also noted in Bernabé et al., 2011). However, in addition to being slightly elongated or flattened (roughly corresponding to prolate or oblate ellipsoids), sand grains have irregular, uneven surfaces that further increase $A_{G}$ and therefore $f_{G}$. Since the Biella and Tabacco (1981) and Biella et al. (1983) sands were described as rounded, we only increased $f_{G}$ by a limited amount. Our final $f_{G}$ estimates ranged from about 1.5 for the coarsest sands to 1.9 for the finest ones. Owing to the relative imprecision of $f_{G}$, we evaluated $\delta r_{H} / r_{H}$ around $\pm 10 \%$ (Fig. 2).

In log-log plots of permeability and formation factor versus the estimated hydraulic radius (Fig. 2), we observe power law dependences of $k$ on $r_{H}$ with exponents equal to 1.91 and 1.96 for glass beads and sand, respectively (notice that the exponent increases to 1.98 when Glover and Walker (2009) three coarsest glass bead packs are omitted to avoid the end effect mentioned in the previous paragraph). Conversely, $F$ displays very weak variations associated with a possible minor correlation of grain size with grain shape and/or packing structure. In summary, well-sorted, unconsolidated granular media represent an excellent approximation of materials possessing a variable length scale $r_{H}$ and constant scale-invariant factors 

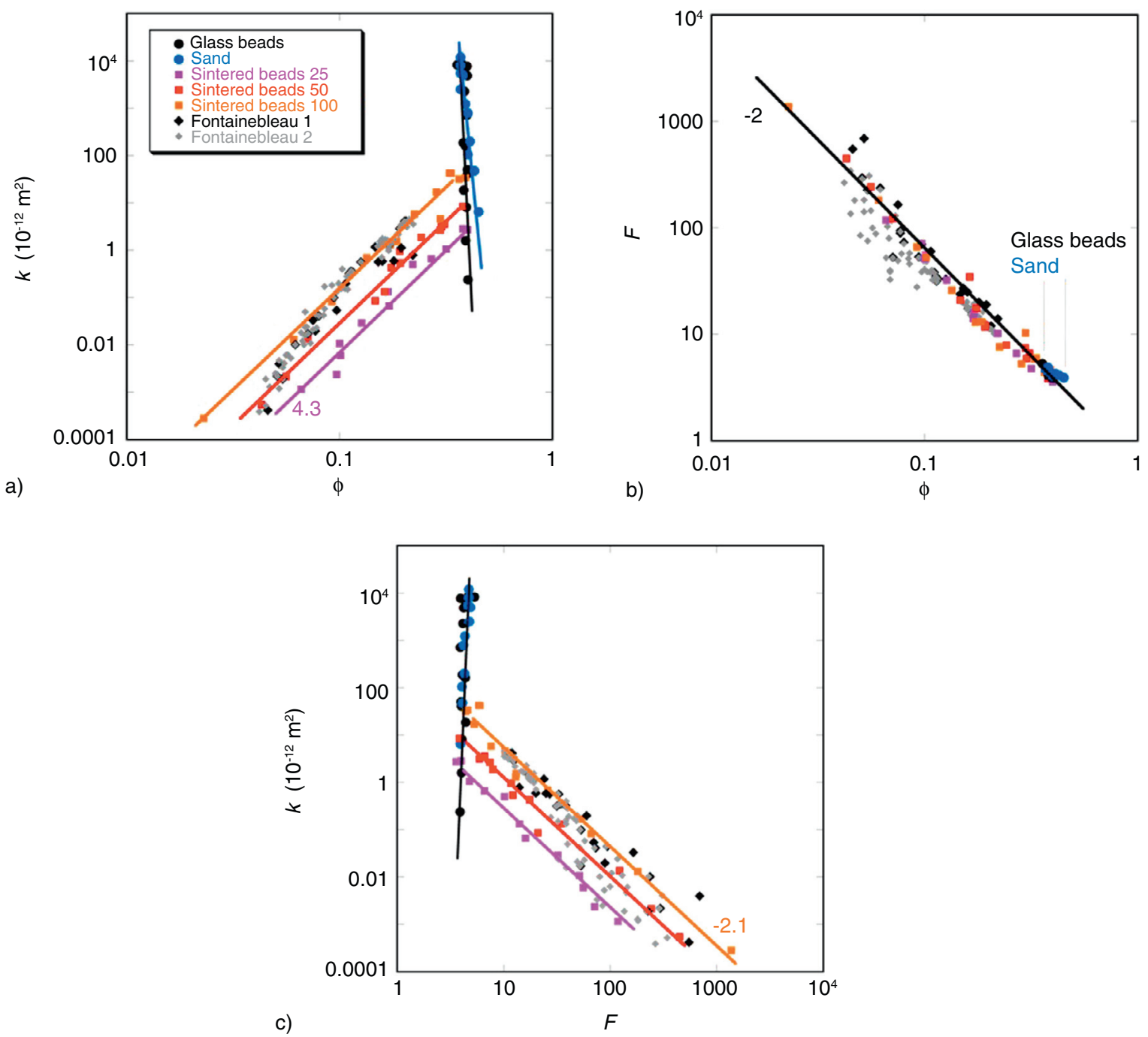

Figure 1

Compilation of published experimental data, a) $k$ versus $\phi$, b) $F$ versus $\phi$ and c) $k$ versus $F$, in unconsolidated monodisperse glass beads (Glover and Walker, 2009; Glover and Déry, 2010; black dots), well-sorted, rounded sands (Biella and Tabacco, 1981; Biella et al., 1983; blue dots), sintered glass beads (Wong et al., 1984; Guyon et al., 1987; Li et al., 1995; Blair et al., 1996; purple, red and orange squares corresponding to different grain sizes as indicated in the inset) and Fontainebleau sandstone (Doyen, 1988; Fredrich et al., 1993; Zamora, unpublished data; black diamonds; Revil et al., 2014; grey diamonds). The colored lines are inserted to highlight power-law trends in the various materials (note that the three families of sintered glass obey fairly similar power laws). The values of the exponents are given when necessary.

(with the possible exception of some minor factors that are not investigated in the present study).

\subsection{Sintered Glass Beads}

Sintering of granular materials is a densification process driven by the excess free energy associated with the surface of the grains. Sintering is activated by raising temperature to a sufficiently high value. Sintered glass beads have often been used as rock proxies to study the effect of porosity on the physical properties of rocks. In particular, Wong et al. (1984), Guyon et al. (1987), Li et al. (1995) and Blair et al. (1996) reported measurements of $k, F$ and $\phi$ in sintered monodisperse glass beads. They used three main types of glass beads with radii of around 25, 50 and $100 \mu \mathrm{m}$ (materials with grain radii as low as $90 \mu \mathrm{m}$ and as high as $160 \mu \mathrm{m}$ are included in the last group). Despite large fluctuations, the three categories display very similar power-law trends of permeability versus $\phi$ and $F\left(k \propto \phi^{4.3}\right.$ and $k \propto F^{-2.1}$; Fig. 1a, c) and of the formation factor with respect to porosity $\left(F \propto \phi^{-2}\right.$, i.e., the classic Archie's relation; Fig. 1b). 

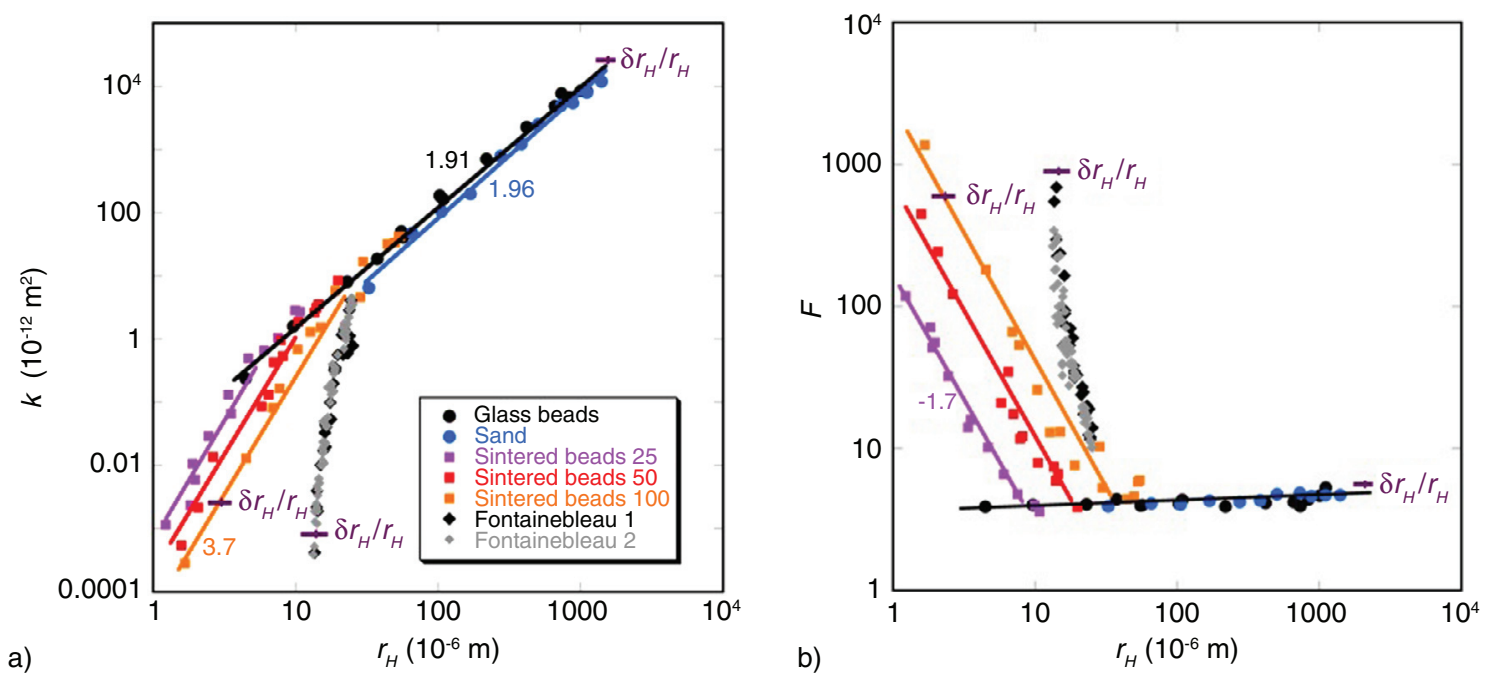

Figure 2

Influence of the hydraulic radius on a) permeability and b) formation factor. The diagrams use the same symbol and line conventions as in Figure 1. Three horizontal error bars (dark purple) help visualize the relative uncertainty $\delta r_{H} / r_{H}$ determined for the various materials (i.e., $\pm 10, \pm 15$ and $\pm 20 \%$ for the unconsolidated sands, sintered glass beads and Fontainebleau sandstone, respectively).

During sintering solid matter is transferred over very short distances and the total volume of solid does not change, even though the density of the aggregate increases. Densification of the granular assembly is caused entirely by redistribution of the solid phase and decrease of the pore volume. This process produces an augmentation of the number of grain contacts per grain very similar to the one experimentally observed in plastically compressed bronze powders (Fischmeister et al., 1978). Using these properties of sintering, we developed a method to estimate the hydraulic radius of monodisperse sintered glass beads from porosity measurements and the initial radius of the spheres (Appendix A for more details). Here, we estimated the relative uncertainty $\delta r_{H} / r_{H}$ to be $\pm 15 \%$ (Fig. 2).

We observe that permeability, formation factor and porosity follow well-defined power laws of the hydraulic radius, $k \propto r_{H}^{3.7}$ (Fig. 2a), $F \propto r_{H}^{-1.7}$ (Fig. 2b) and $\phi \propto r_{H}^{0.9}$ (not shown). The observed exponents are substantially different from those predicted by the PWB and Bernabé et al. models (Eq. 1-6) when the scale-invariant factors are assumed constant. These differences imply that, during sintering, variations in hydraulic radius were coupled with changes in the major scale-invariant factors, in particular pore connectivity.

\subsection{Fontainebleau Sandstone}

Fontainebleau sandstone is a $99 \%$ pure quartz arenite from the Paris basin, France. It is relatively well sorted with a mean grain radius of about $120 \mu \mathrm{m}$. One characteristic that makes Fontainebleau sandstone an excellent material for testing rock physics models, is that the formation includes a very wide range of porosities (from as low as 0.03 to about 0.25). In a seminal study, Bourbié and Zinszner (1985) measured permeability, porosity and the acoustic properties of a large set of Fontainebleau sandstone specimens. Moreover, they performed a detailed microstructure investigation using a variety of techniques, including the preparation of pore casts. Similarly, Doyen (1988) and Fredrich et al. (1993) combined quantitative microstructure observations and laboratory measurements of $k, F$ and $\phi$. They used twodimensional images of the pore structure to estimate the hydraulic radius (Fredrich et al., 1993), the mean pore coordination number $z$ and the mean pore radius $\langle r\rangle$ (Doyen, 1988). The statistical distributions of porosity, pore coordination number $z$, throat radius $r$ and pore length $l$ were measured in three-dimensional images of several Fontainebleau sandstone samples by Lindquist et al. (2000). Furthermore, we complemented the datasets cited above with the laboratory measurements of $\phi, k$ and $F$ (including surface conduction correction) of Revil et al. (2014) and Zamora (unpublished data).

As reported by Bourbié and Zinszner (1985), Fontainebleau sandstone displays a very well defined relationship between porosity and permeability (Fig. 1a). It is then reasonable to assume that other transport properties and microstructural quantities may also be accurately expressed as functions of porosity, allowing the individually 
TABLE 2

The transforms used to complete the Fontainebleau sandstone datasets of Doyen (1988), Fredrich et al. (1993), Revil et al. (2014) and Zamora (unpublished data)

$l=73.284 \phi^{-0.39752}$

Lindquist et al. (2000)

$r_{\mathrm{H}}=9.528+94.705 \phi-104.94 \phi^{2}$

Doyen (1988), Fredrich et al. (1993) and Lindquist et al. (2000)

$z=10.366+6.2455 \log _{10} \phi$

Doyen (1988) incomplete datasets of Doyen (1988), Fredrich et al. (1993), Revil et al. (2014) and Zamora (unpublished data) to be blended together into a more complete one (for example, see the approach used by Bernabé et al., 2010). However, visual inspection shows that the formation factor values reported in Revil et al. (2014) are significantly lower and more scattered than the other data sets (as is particularly evident for the samples with $\phi<0.12$; Fig. 1b, c). This discrepancy is probably not caused by surface conduction since the presumably uncorrected formation factors of Doyen (1988) and Fredrich et al. (1993) are actually higher than the corrected ones, while the reverse would be expected if surface conduction played a major role. Moreover, note that Doyen (1988) and Fredrich et al. (1993) used highly saline $\mathrm{NaCl}$ solutions, which cause negligible surface conduction in clay-free rocks such as Fontainebleau sandstone.

In order to acknowledge the differences in $F$, we divided the data into two groups, Fontainebleau 1 containing Doyen (1988), Fredrich et al. (1993) and Zamora (unpublished data) data, and Fontainebleau 2 including only the Revil et al. (2014) data. The two groups of $(k, \phi)$ data points appear well superposed on a log-log scatterplot (Fig. 1a) and display the classic downward-curved trend originally reported by Bourbié and Zinszner (1985). We also note that the Fontainebleau sandstone data approximately coincide with those of the coarsest sintered glass beads, as should be expected since they nearly have the same grain size. The formation factor of the Fontainebleau 1 group is consistent with the classic Archie's relation, $F \propto \phi^{-2}$, while a smaller cementation exponent of about 1.8 is observed for the Fontainebleau 2 group (Fig. 1b). The difference between the Fontainebleau 1 and 2 groups is also visible in Figure 1c, where permeability is represented as a function of formation factor.

Bernabé et al. (2010) constructed a functional transform to calculate $r_{H}$ as a function of $\phi$ in Fontainebleau sandstone based on Fredrich et al. (1993) results. This transform was established using only 4 data points and, therefore, may have limited applicability. Here, we tried to construct a more robust $r_{H}(\phi)$ transform (Tab. 2) by assuming that the average pore and/or throat radius values reported by Doyen (1988) and Lindquist et al. (2000) could be used as hydraulic radius proxies. Notice that the relative difference of the two transforms is modest, between 2 and $8 \%$, and that the new one has a slightly greater range of variation, corresponding to a relative uncertainty $\delta r_{H} / r_{H}$ of about $\pm 20 \%$.

We observe that permeability declines much more rapidly with decreasing $r_{H}$ in Fontainebleau sandstone than in the sintered glass beads (Fig. 2a) whereas the formation factor increases more sharply (Fig. 2b, in which the difference between Fontainebleau 1 and Fontainebleau 2 is visible). The implication is that the evolution of the pore structure during diagenesis of Fontainebleau sandstone (mostly quartz cementation) differs from that during sintering.

\section{DATA ANALYSIS AND MODELING}

The procedure for testing the PWB model is as follows. According to a strict geometrical interpretation of PWB, we assign fixed constant values to the input parameters, $D=0.1$ and $\tau^{2}=2$. We then calculate the PWB predictions of $k$ and $F$ using Equations (1-3) and compare the results to the experimental data. The model is deemed acceptable if the calculated $k$ and $F$ values fall within some intervals $\pm \delta k$ and $\pm \delta F$, respectively, of the corresponding experimental values. Since $D$ and $\tau^{2}$ are not allowed to vary, the model tolerances are given by $\delta k / k \approx 2 \delta r_{H} / r_{H}+\delta \phi / \phi$ and $\delta F / F \approx \delta \phi / \phi$, where the $\delta \phi / \phi$ term is small compared to $\delta r_{H} / r_{H}$.

We specifically chose this procedure to test whether or not a model exclusively sensitive to variations in the characteristic length scale $r_{H}$ could explain the experimental data. If a different goal were pursued, flexibility could be increased by separately considering the hydraulic and electrical tortuosities, $\tau_{h}{ }^{2}=D \phi r_{H}{ }^{2} / k$ and $\tau_{e}{ }^{2}=\phi F$. In this second approach, the effects of scale-invariant factors such as pore connectivity, are implicitly incorporated into $\tau_{h}{ }^{2}$ and $\tau_{e}{ }^{2}$. Note that, in order to be consistent with Equations (1) and (2), Equation (3) requires formal equality of $\tau_{h}^{2}$ and $\tau_{e}^{2}$ but it does not exclude large variations of tortuosity as a function of $\phi$.

Similarly, in order to test the Bernabé et al. (2011) model, we need to assign input values to the mean pore coordination number, $z$, the normalized pore length, $l / r_{H}$, the heterogeneity measure, $\sigma$, and the cross-sectional aspect ratio, $\varepsilon$. For this purpose, we prefer to use actual measurements if they exist (a rare case). When direct experimental data are not available, the best option is to infer the values of the desired parameters from measurements of other related quantities. The inference scheme may be based on a theoretical model (e.g., the estimation of $r_{H}$ for unconsolidated glass beads using Eq. 8) or on a purely empirical relationship (e.g., the $r_{H}(\phi)$ transform established for Fontainebleau sandstone). When even indirect information is unavailable, the last option is to identify plausible, reasonably narrow 
TABLE 3

Summary of the values assigned to the input parameters of the Bernabé et al. models

\begin{tabular}{|c|c|c|c|c|}
\hline & $z$ & $\sigma$ & $l / R_{G}$ & $\varepsilon$ \\
\hline $\begin{array}{l}\text { Unconsolidated glass } \\
\text { beads }\end{array}$ & 6 & 0.45 & 1.3 & 1 \\
\hline Unconsolidated sands & 6 & 0.45 & 0.85 & 1 \\
\hline Sintered glass beads & $\begin{array}{c}\text { Linear } \\
6(\phi=0.4) \\
4(\phi=0.02)\end{array}$ & $\begin{array}{c}\text { Linear } \\
0.45(\phi=0.4) \\
0.7(\phi=0.02)\end{array}$ & $\begin{array}{c}\text { Linear } \\
1.3(\phi=0.4) \\
0.4(\phi=0.02)\end{array}$ & 1 \\
\hline Fontainebleau sandstone & $\begin{array}{c}\text { Logarithmic } \\
6(\phi=0.2) \\
1.6(\phi=0.04)\end{array}$ & 0.75 & $\begin{array}{c}\text { Power law } \\
1.2(\phi=0.2) \\
2.2(\phi=0.04)\end{array}$ & $\begin{array}{c}\text { Linear } \\
0.3(\phi=0.2) \\
0.03(\phi=0.04)\end{array}$ \\
\hline
\end{tabular}

ranges of variation of the input parameters and test the models using values falling within these ranges.

Examination of the functions $C_{k}(\sigma, \varepsilon), C_{F}(\sigma, \varepsilon), \beta(\sigma)$ and $\gamma(\sigma)$ in Equations (4) and (5) shows that the Bernabé et al. (2011) model is more sensitive to $\sigma$ and $z$ than $l / r_{H}$ and $\varepsilon$. Furthermore, the sensitivity of the model strongly depends on the magnitude of $\sigma$ and $z$. For example, imposing a change $\delta z= \pm 0.1$ (or, equivalently, $\delta \sigma= \pm 0.05$ ) results in a relative variation of calculated $k$ as low as $\pm 1 \%$ for $\sigma=0.05$ and $z=10$ and as high as $\pm 60 \%$ for $\sigma=0.95$ and $z=2$ (moderately smaller relative variations are obtained for $F$ in the same conditions).

\subsection{Unconsolidated Glass Beads and Sands}

It is rather obvious that unconsolidated monodisperse bead packs and well-sorted sands have a well-connected pore space, for which the appropriate value of $z$ is on the order of 6 , and a relatively homogeneous pore structure with $\sigma$ between 0.3 and 0.5 (Bernabé et al., 2011, and references therein). It is also clear that the cross-sections of the pores, although not circular, are not elongated in a particular direction; thus, $\varepsilon$ should be taken to be unity (Bernabé et al., 2011). Finally, the average pore length, $l$, must be related to the grain radius. Bernabé et al. (2011) estimated $l \approx 1.2 R_{G}$ for a Face Centered Cubic (FCC) array of identical spheres, but random sphere packs are less dense than FCC and a slightly greater value may therefore be more realistic (here, we used $l=1.3 R_{G}$ ). In sand packs, the situation is different. We must account for a relatively broad grain size distribution and recognize that, in the average, the pore length should be more strongly controlled by the fine particles than be the coarse ones (see the analysis of binary sand mixtures in Bernabé et al., 2011). Well-sorted sands have grain radius distributions with a standard deviation lower than about $1 / 3$ of the mean grain radius and hence contain a significant fraction of grains with a radius as low as half of the mean grain radius. Thus, values of $l$ as low as $0.65 R_{G}$ can reasonably be assumed (here, we used $l=0.85 R_{G}$ ). For convenience, the assigned values of the input parameters are summarized in Table 3 .

Owing to moderately low $\delta r_{H} / r_{H}$, high $z$ and low $\sigma$, the $\delta k / k$ and $\delta F / F$ tolerances for both models were found to be relatively small, i.e., $\delta k / k< \pm 30 \%$ and $\delta F / F< \pm 10 \%$. Despite these relatively tight tolerances, both the PWB and Bernabé et al. models fitted the experimental $k$ and $F$ data quite well for both glass bead packs and sands (Fig. 3). We note, however, a slight tendency of the models (especially, when expressed in Eq. 3 and 6) to overestimate the permeability of the glass-bead packs (Fig. 3a, c). It is also worth emphasizing that a strictly geometrical definition of tortuosity (i.e., $\tau^{2}=2$ ) is sufficient to ensure a good fit of the PWB model.

\subsection{Sintered Glass Beads}

Clearly, sintering is bound to produce significant structural changes in the glass bead packs. We expect pore connectivity to decrease and pore-scale heterogeneity to increase during sintering. Also the reduction of the intergranular distances should lead to a simultaneous decrease of the pore length. One of our main assumptions in this paper is that structural changes accompanying processes such as sintering or diagenesis, should be gradual and that the model parameters should be expressible as simple, continuous functions of porosity. Here, for the sake of simplicity, we limited our analysis of $z, \sigma$ and $l$ to linear functions of $\phi$. At very low levels of sintering (i.e., high porosity, $\phi=0.4$ ), $z, \sigma$ and $l$ should evidently take the values mentioned in Section 3.1 for unconsolidated glass bead packs (i.e., $z=6, \sigma=0.45$ and $\left.l=1.3 R_{G}\right)$. To determine the linear functions $z(\phi)$, $\sigma(\phi)$ and $l(\phi)$ we only need to find the adequate values at the low porosity end-member. Having no microstructure information on the sintered glass beads at low porosities, we proceeded by trial and error and found that $z=4$, $\sigma=0.7$ and $l=0.4 R_{G}$ at $\phi=0.02$, yielded satisfactory results. The assigned values of the input parameters are summarized in Table 3. 

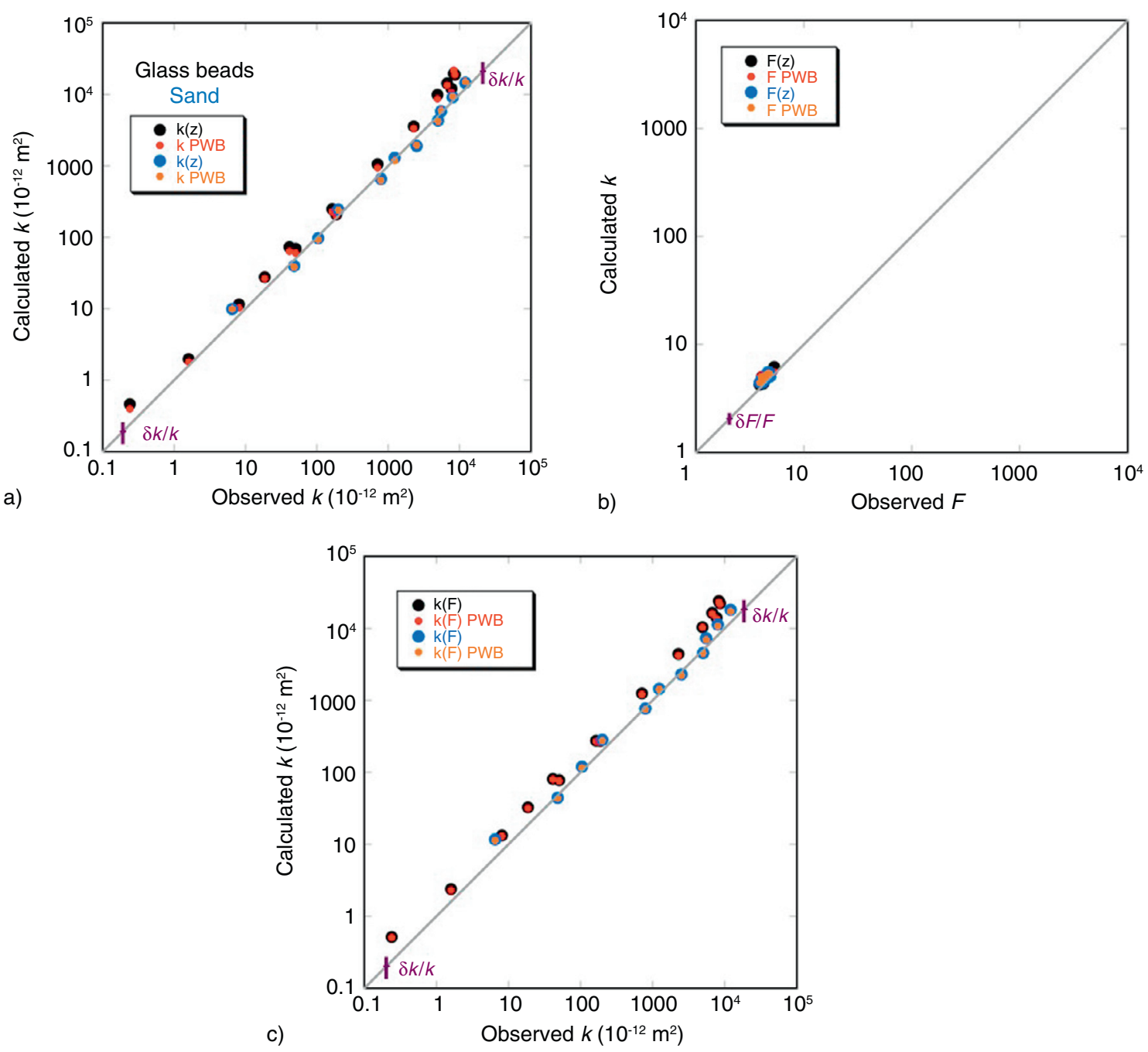

Figure 3

Comparison of calculated a) $k$ from Equations $(1,4)$, b) $F$ from Equations $(2,5)$ and c) $k$ from Equations $(3,6)$ to the observed values of permeability and formation factor in the unconsolidated glass beads and sands. The meaning of the different symbols is explained in the inset, where $k(z)$ refers to Bernabé et al. Equation (4), $F(z)$ to Equation (5) and $k(F)$ to Equation (6). The vertical error bars (dark purple) represent the tolerances estimated for the Bernabé et al. model, $\delta k / k \approx \pm 30 \%$ and $\delta F / F \approx \pm 10 \%$.

The main result is that, in sintered glass beads, the Bernabé et al. Equations $(4,5)$ worked significantly better than the PWB Equations $(1,2)$ (Fig. 4a, b). As expected from the results described in Section 3.1, the two models gave almost identical, well-matching results at porosities near 0.4 but PWB produced increasingly strong overestimation of $k$ and underestimation of $F$ with decreasing porosity. The $\delta k / k$ and $\delta F / F$ tolerances calculated for the PWB model were clearly too small to explain the observed discrepancies of up to one order of magnitude at very low $\phi$. However, the tolerances for both models were nearly sufficient to account for the experimental sample-to-sample variability. Surprisingly, we found identically good fits with both the PWB
Equation (3) and Bernabé et al. Equation (6) (Fig. 4c). The success of Equation (3) is remarkable since this equation was derived from Equations $(1,2)$, which both appear to be invalid in sintered glass beads. This outcome gives support to the classic idea of using electrical conductivity measurements to estimate the "connectedness" of the pore space and incorporate it into a permeability model (Revil and Cathles, 1999; Glover et al., 2006; Glover, 2009).

\subsection{Fontainebleau Sandstone}

Based on the reported microstructural data (Doyen, 1988; Fredrich et al., 1993; Lindquist et al., 2000), Bernabé 

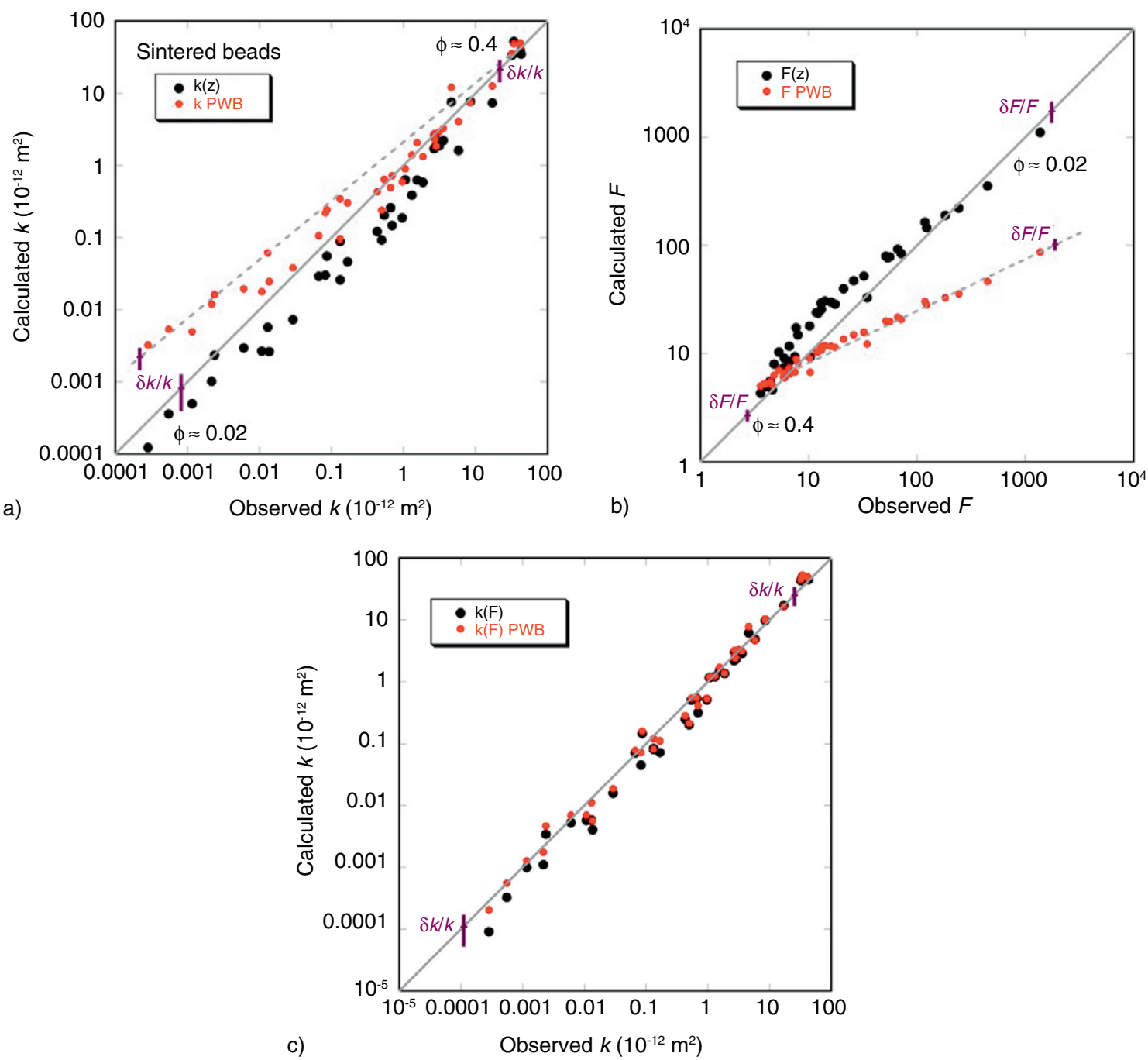

Figure 4

Comparison of calculated a) $k$ from Equations $(1,4)$, b) $F$ from Equations $(2,5)$ and c) $k$ from Equations $(3,6)$ to the observed values of permeability and formation factor in the sintered glass beads. The meaning of the different symbols is explained in the inset, where $k(z)$ refers to Bernabé et al. Equation (4), $F(z)$ to Equation (5) and $k(F)$ to Equation (6). The vertical error bars (dark purple) represent minimal estimates of the model tolerances (i.e., $\delta k / k \approx \pm 30 \%$ and $\delta F / F \approx \pm 10 \%$ for the PWB model as well as the Bernabé et al. model applied to high porosity samples; $\delta k / k \approx \pm 50 \%$ and $\delta F / F \approx \pm 20 \%$ for the Bernabé et al. model in low porosity samples).

et al. $(2010,2011)$ obtained approximate expressions of the pore length $l$ and pore coordination number $z$ as functions of porosity (Tab. 2). These transforms describe a large reduction of $z$ from about 6 to 1.6 (very near the percolation threshold) and a substantial increase of $l / R_{G}$ from 1.2 to 2.2 , over a wide range of porosities (i.e., from 0.2 to 0.04 ). Bernabé et al. $(2010,2011)$ argued that pore space images (Bourbié and Zinszner, 1985; Doyen, 1988) suggest that the pores in Fontainebleau sandstone tend to have elongated cross-sections and that the cross-sectional aspect ratio $\varepsilon$ decreases with decreasing porosity. The fact that the highest porosities reported in Doyen (1988), Fredrich et al. (1993),
Revil et al. (2014) and Zamora (unpublished data) were much lower than 0.4 , the porosity of unconsolidated sands, suggests that the corresponding rock samples had already experienced a significant amount of diagenesis and were therefore likely to have pore aspect ratios lower than unity. For the sake of simplicity, we assumed that $\varepsilon$ was a linear function of $\phi$, decreasing from 0.31 to 0.03 in the porosity range mentioned above. The pore cross-section area and pore length data reported by Lindquist et al. (2000) approximately satisfied highly skewed exponential distributions, which have equal standard deviations and means, suggesting that the heterogeneity measure $\sigma$ should also be relatively 

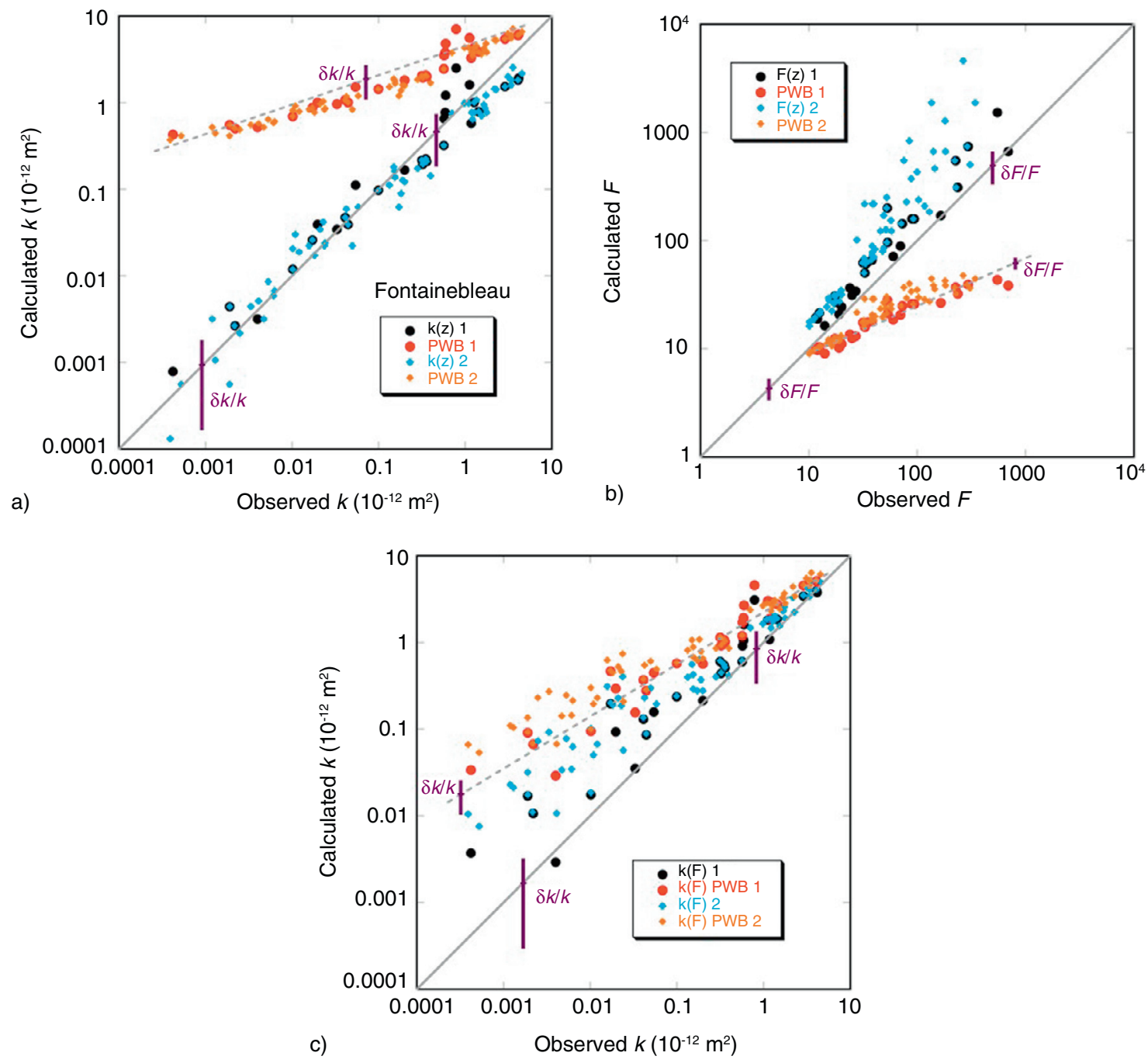

Figure 5

Comparison of calculated a) $k$ from Equations $(1,4)$, b) $F$ from Equations $(2,5)$ and c) $k$ from Equations $(3,6)$ to the observed values of permeability and formation factor in the Fontainebleau sandstone. The meaning of the different symbols is explained in the inset, where $k(z)$ refers to Bernabé et al. Equation (4), $F(z)$ to Equation (5) and $k(F)$ to Equation (6) and where the digits 1 and 2 indicate the Fontainebleau 1 and 2 datasets. The difference between the two datasets is particularly visible in the diagrams $b$ ) and c). The vertical error bars (dark purple) represent minimal estimates of the $\delta k / k$ and $\delta F / F$ model tolerances (i.e., $\delta k / k \approx \pm 40 \%$ and $\delta F / F \approx \pm 10 \%$ for the PWB model; $\delta k / k \approx \pm 60 \%$ and $\delta F / F \approx \pm 20 \%$ for the Bernabé et al. model in high porosity samples and $\delta k / k \approx \pm 90 \%$ and $\delta F / F \approx \pm 30 \%$ in low porosity samples).

high. By trial and error, we found that a constant value $\sigma=0.75$ gave satisfactory results. The assigned values of the input parameters are summarized in Table 3.

The results obtained for the Fontainebleau sandstone datasets are quite similar to those for the sintered glass beads. The PWB Equations (1-3) yielded adequate results only for the samples with highest porosities but overestimated $k$ by up to three orders of magnitude and underestimated $F$ by one and half at the lowest porosities (Fig. 5). The observed discrepancies were much greater than the $\delta k / k$ and $\delta F / F$ tolerances calculated for the PWB model.
In contrast, Bernabé et al. Equation (4) produced a very good fit with data for both Fontainebleau 1 and Fontainebleau 2 (Fig. 5a). Equation (5) was also satisfactory, although the estimated $\delta F / F$ tolerance could not offset the large overestimation of $F$ observed for the low porosity samples of the Fontainebleau 2 datasets (Fig. 5b). Equation (6), while still relatively acceptable, displayed a visibly poorer fit quality than Equation (4), particularly for the Fontainebleau 2 dataset (Fig. $5 \mathrm{c}$ ). The data scatter associated with both the PWB Equation (3) and Bernabé et al. Equation (6) was much larger in Fontainebleau sandstone (even if we only 


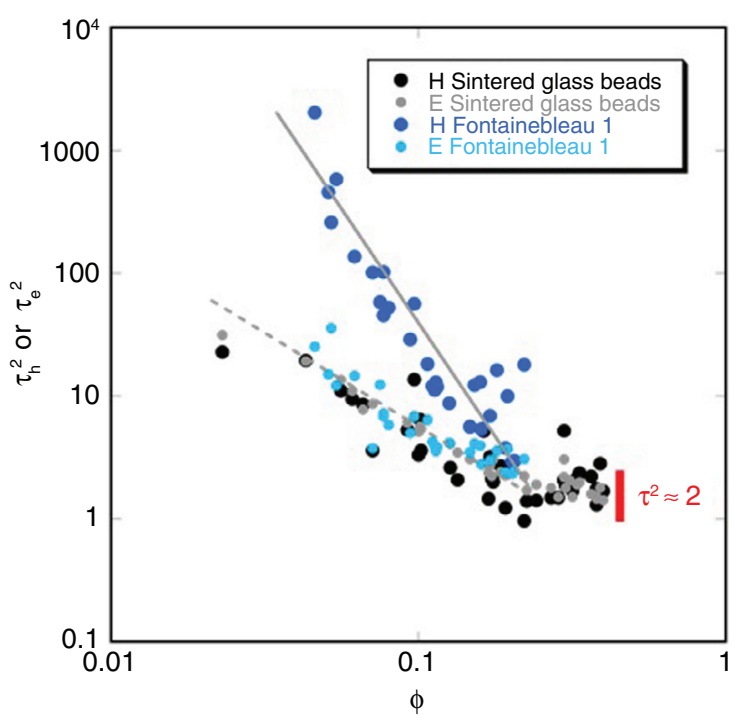

Figure 6

The hydraulic and electrical tortuosities $\tau_{h}^{2}$ and $\tau_{e}^{2}$ versus porosity for the sintered glass beads and Fontainebleau sandstone. The meaning of the different symbols is explained in the inset, where $\mathrm{H}$ and $\mathrm{E}$ stand for hydraulic and electrical. For the sake of visibility, the Fontainebleau 2 dataset is not included in this diagram but the associated trends are comparable to the Fontainebleau 1 trends represented here.

consider the Fontainebleau 1 dataset) than in the sintered glass beads (compare Fig. $4 \mathrm{c}$ and $5 \mathrm{c}$ ), suggesting that additional, relatively important scale-invariant factors were probably omitted in the models.

\section{DISCUSSION}

The main observation reported here is that the PWB model correctly predicts the transport properties of unconsolidated, well-sorted granular media (Fig. 3) but becomes gradually more inexact when pore connectivity is altered by processes such as sintering or cementation-controlled diagenesis (Fig. 4, 5). Our task in this discussion is to identify the origin of the PWB breakdown.

The granular materials investigated by Biella and Tabacco (1981), Biella et al. (1983), Glover and Walker (2009) and Glover and Déry (2010) cover nearly three orders of magnitude in grain size and nevertheless display very narrow ranges of porosity ( 0.37 to 0.41 , except a couple of outliers as high as 0.45 ) and formation factor (4 to 5). This insensitivity to grain size demonstrates that these materials have essentially identical pore structures, characterized by nearly constant scale-invariant factors. Their permeability, therefore, must almost exclusively depend on $r_{H}$, the length scale

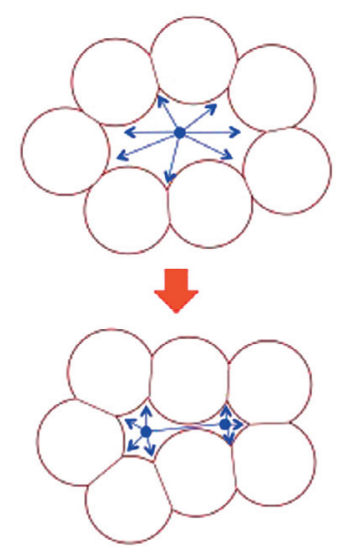

a)

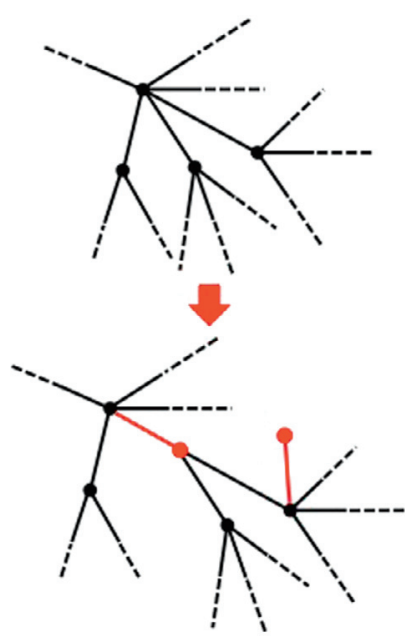

b)

Figure 7

a) Schematic representation of the node splitting mechanism. The cartoon is two-dimensional but it is meant to illustrate a three-dimensional arrangement of sintered grains and pores between them. The large blue dots indicate the center of the nodes and the blue arrows symbolize the (out of plane) channels emanating from them. The large red arrow indicates the evolution associated with sintering. b) Example of graph transformations that leave the graph topological invariant (genus) unchanged while reducing the mean coordination number. In this cartoon, extra node/branch combinations (represented in red) are inserted at different places in the graph without altering the genus. By definition, nodes must always be present at the extremities of a branch (the dotted segments are intended to symbolize the unspecified continuation of the graph).

considered here. As discussed in Section 2.1, Equation (8) allows an accurate estimation of $r_{H}$ for glass bead packs. For the sands, Equation (9) is satisfactory, although the asphericity factor $f_{G}$ is somewhat uncertain. Moreover, good fits were generally obtained with both the PWB and Bernabé et al. models using realistic values of the input parameters. Accordingly, we can conclude that these data supports both the PWB and Bernabé et al. models with constant scaleinvariant parameters, summarized in the following power laws, $k \propto r_{H}^{2}$ and $F \propto r_{H}^{0}$.

In sintered glass beads and Fontainebleau sandstone, on the other hand, the predictions of Equations $(1,2)$ (denoted $k_{P W B}$ and $F_{P W B}$, respectively) become strongly erroneous (Fig. 4, 5). As mentioned earlier, another way to look at Equations $(1,2)$ is to calculate the hydraulic and electrical tortuosities $\tau_{h}^{2}$ and $\tau_{e}^{2}$. Both parameters are found to increase with decreasing porosity (Fig. 6). One interesting observation is that $\tau_{h}^{2}$ and $\tau_{e}{ }^{2}$ have comparable values in sintered glass beads (consistent with the validity of Eq. 3 demonstrated by Fig. 4c), whereas $\tau_{h}^{2}$ increases much faster than 
$\tau_{e}^{2}$ in Fontainebleau sandstone (Fig. 5c). Furthermore, the rate of increase of $\tau_{e}^{2}$ is about the same for both materials (Fig. 6). As a quantitative summary, we note that, at porosities of about 0.02 to 0.04 , the ratio $k_{P W B} / k$ reached values as low as $1 / 10$ and $1 / 1000$ in sintered glass beads and Fontainebleau sandstone, respectively, while $F_{P W B} / F$ approached 15 in both materials. What causes these discrepancies? PWB has three easily identified unrealistic assumptions, (a) the pores have idealized shapes, namely, straight cylinders or flat slits, (b) the pore space is fully connected and pore connectivity cannot change, (c) fluctuations in pore size, length and other geometric characteristics are negligible.

We will first discuss hypothesis (a). The very small range of variation of the form factor $D$ mentioned in Section 1 suggests that the cross-sectional shape of the pores does not have a substantial effect on fluid flow (indeed, square and equilateral triangular conduits have form factors of 1/9 and 1/9.6, respectively). Although complex cross-sectional shapes such as stars with narrow spokes may produce a greater effect (Yale, 1984), unrealistic cross-sectional pore shapes can be ruled out as the main cause of the breakdown of PWB in sintered glass beads and Fontainebleau sandstone.

Alternatively, we note that channels in porous rocks have constricted shapes with smaller cross-sections (usually known as throats) in their middle parts than at the ends. A gradual narrowing of the constrictions during sintering and diagenesis will increasingly impede fluid flow and may explain the breakdown of PWB. In order to estimate this effect, we calculated the hydraulic and electrical conductances of a frustum-shaped pipe (i.e., truncated cone) and compared them to those of an equivalent straight cylindrical pipe (Appendix B).

In order to achieve identical values of porosity and hydraulic radius, the frustum and cylindrical pipes must have identical volumes and wetted surface areas, yielding the following equations:

$$
\begin{aligned}
& 3 l r_{H}^{2}=l_{c}\left(r_{2}^{2}+r_{1} r_{2}+r_{1}^{2}\right) \text { and } \\
& 2 l r_{H}=\left(r_{1}+r_{2}\right) \sqrt{\left(r_{2}-r_{1}\right)^{2}+l_{c}^{2}}
\end{aligned}
$$

where $r_{1}, r_{2}>r_{1}$ and $l_{c}$ denote the end-face radii and length of the frustum pipe, and, $r_{H}$ and $l$ refer to the estimated pore radius and pore length discussed in Sections 2 and 3. Although intuitively appealing, assuming $l_{c}=l$ is not practical since the system of Equations (10) does not necessarily admit positive, real solutions for $r_{1}$ and $r_{2}$ besides the obvious but irrelevant $r_{1}=r_{2}=r_{H}$. Alternatively, we can complement the system with either Equations (B5) or (B7) of Appendix $\mathrm{B}$, in which $g_{H}$ is assumed equal to the ratio $k / k_{P W B}$ and $g_{E}$ to $F_{P W B} / F$. We then solve both expanded systems for $r_{1}, r_{2}$ and $l_{c}$, and check whether or not the two sets of solutions are consistent with each other.
For the sintered glass beads, the lowest porosity $(\phi \approx 0.02)$ occurred in the coarsest group $\left(R_{G}=100 \mu \mathrm{m}\right)$, corresponding to $r_{H} \approx 1.7 \mu \mathrm{m}, l \approx 39 \mu \mathrm{m}, g_{H} \approx 1 / 10$ and $g_{E} \approx 1 / 15$. The system with Equation (B5) and that with (B7) produced significantly different values for the constriction radius, i.e., $r_{1} \approx 0.6 \mu \mathrm{m}$ and $0.1 \mu \mathrm{m}$, respectively, although the single values $r_{2} \approx 2.5 \mu \mathrm{m}$ and $l_{c} \approx 45 \mu \mathrm{m}$ were obtained in both cases. The failure to find consistent solutions for $r_{1}$ is due to the fact that $g_{H}$ and $g_{E}$ have comparable magnitudes although a given constriction has a greater effect on fluid flow than electrical conduction. Thus, tightening of pore constrictions is not a plausible cause of the breakdown of PWB in sintered glass beads.

For the Fontainebleau sandstone, at low porosities $(\phi \approx 0.04)$ we have $r_{H} \approx 14 \mu \mathrm{m}, l \approx 250 \mu \mathrm{m}, g_{H} \approx 1 / 1000$ and $g_{E} \approx 1 / 15$, which yield consistent solutions, i.e., $r_{1} \approx 0.9 \mu \mathrm{m}, r_{2} \approx 21 \mu \mathrm{m}$ and $l_{c} \approx 320 \mu \mathrm{m}$, for the two systems. Nevertheless, pore constrictions can be ruled out as major PWB breakdown factors in Fontainebleau sandstone also because $r_{1} \approx 0.9 \mu \mathrm{m}$ is much smaller than Lindquist et al. (2000) reported mean throat radii (from $23 \mu \mathrm{m}$ at a porosity of 0.22 to $18 \mu \mathrm{m}$ at 0.075 ). Notice that, given the very strong throat size heterogeneity observed in Fontainebleau sandstone, we cannot conclude that very narrow constrictions do not exist in this rock and that throat tightening does not happen at all. Our conclusion is merely that throat tightening cannot alone explain the breakdown of the PWB model.

We are left with hypotheses (b) and (c), which are addressed in Bernabé et al. (2010, 2011). We saw in Sections 3.2 and 3.3 that sets of reasonable values of $z, l / r_{H}, \sigma$ and $\varepsilon$ producing good fits with the experimental data, can be found for both the sintered glass beads and Fontainebleau sandstone. These results suggest that sintering and cementation-controlled diagenesis of unconsolidated granular media lead to reduced pore connectivity and increased pore scale heterogeneity. In Fontainebleau sandstone, diagenesis is also associated with flattening of the pores.

One important question is: how does reduction of connectivity occur? As proposed by Zhu et al. (1995, 1999), one possible mechanism is pinching off of conduits, which transforms connecting conduits into pairs of dead-ends and strings of isolated pores. The specific pinching off mechanism invoked by Zhu et al. (1999) is tube ovulation, a surface tension-controlled process that occurs increasingly rapidly with decreasing tube radius. Pinching off should, therefore, be primarily active during the late stages of sintering and diagenesis when appropriately narrow throats are present.

We can also expect re-arrangement of grains to take place during the early stages of sintering and diagenesis, owing to the sufficiently large pore space available for grain motion and to the presence of intergranular mechanical forces 
(gravity and surface tension in the case of sintering and overburden pressure for diagenesis). Such a grain re-arrangement event is schematically illustrated in Figure 7a. The large nodal pore in the upper cartoon is split into two interconnected smaller cavities, each one acting as a new nodal pore and connected to a lower number of conduits than the original one. Hence, splitting of nodal pores is associated with a decrease of the pore coordination number and, therefore, of connectivity (Glover and Walker, 2009, also attributed connectivity loss to grain re-arrangements). Notice that, in the schematic example of Figure $7 \mathrm{a}$, the coordination number reduction is not associated with a change in topology. In graph theory, the appropriate topological invariant is the first Betti number (or genus), $\zeta=N_{b}-N_{n}+N_{c}$, where $N_{b}$ denotes the number of branches, $N_{n}$ the number of nodes and $N_{c}$ the number of separate connected clusters (in Fig. $\left.7 \mathrm{a}, N_{c}=1\right)$. Indeed, $\zeta$ is left unchanged by the grain re-arrangement event represented in Figure $7 \mathrm{a}$ whereas the mean coordination number $z$ decreases (see also the example of Fig. 7b, where branch-node pairs are added at diverse locations without changing $\zeta$ ).

As argued by Bernabé and Maineult (2015), pore connectivity is not a pure topological property. In network simulations, the dissipation of energy associated with fluid flow occurs in the branches while the nodes are just locations were mass conservation is enforced. If the resistance of a particular branch is negligibly small, this branch can be removed and the two nodes connected to it merged together without any change in the numerical results (Bernabé and Maineult, 2015). Numerical merging of nodes was indeed found to be necessary in three-dimensional microstructure studies using skeletonization algorithms, which tend to define nodes only at triple junctions $(z=3)$ and blind ends $(z=1)$. These algorithms thus identify many hydraulically irrelevant branches and require implementation of corrective steps to eliminate them. For example, Lindquist et al. (2000) devised a rule to merge nodes connected by branches shorter than some threshold value. Similarly, Petford et al. (2001) measured $z$ by considering only the branches possessing a significant constriction (in other words, by counting the throats) and by merging the redundant nodes. The Bernabé et al. $(2010,2011)$ model, of course, assumes hydraulically representative values of $z$ (for example, they found that the $z(\phi)$ transform based on Doyen's, 1988, data gave better results that the one from Lindquist et al., 2000).

In summary, the processes referred to above as pinching off and node splitting could explain the regular reduction in pore connectivity inferred from the sintered glass beads and Fontainebleau sandstone experimental data. Although they both reduce the mean pore coordination number, these two processes affect the pore space topology differently. Pinching off of a conduit adds two nodes and a branch and hence decreases $\zeta$ by one. In the example of Figure 7a, node splitting leaves $\zeta$ constant, but, if a node splits into more than two connected nodes and if the new nodes form a loop, the genus $\zeta$ will increase. Therefore, determining the genus of the skeletonized pore space of rock samples may provide useful insight into the rock genetic processes, although we do not expect $\zeta$ (unlike the coordination number $z$ ) to be formally related to the rock transport properties.

\section{CONCLUSIONS}

Our main conclusions are:

- one of the most important scale-invariant factors controlling permeability is pore connectivity;

- a recent permeability model that explicitly includes the effect of connectivity and takes connectivity variations into account, performs much better than an older, widely used one that does not;

- the application of the Bernabé et al. model indicates that pore connectivity was continuously and gradually reduced during sintering and cementation-controlled diagenesis;

- examination of the sintered glass beads and Fontainebleau sandstone experimental data also suggests that poreplugging processes (e.g., pinching off) should be active only during the late stages of sintering and diagenesis, and should be complemented by other processes during the early stages. For example, grain re-arrangements occurring in highly porous samples may have lead to the division of large nodal pores into smaller, less connected ones, thus causing the pore coordination number to decrease.

\section{ACKNOWLEDGMENTS}

This work was partially funded by the Open Fund PLN1501 of the State Key Laboratory of Oil and Gas Reservoir Geology and Exploitation (Southwest Petroleum University, Chengdu, China) and by the U.S. Department of Energy under grant DE-FG09-97ER14760.

\section{REFERENCES}

Bernabé Y., Li M., Maineult A. (2010) Permeability and pore connectivity: a new model based on network simulations, J. Geophys. Res. 115, B10203.

Bernabé Y., Zamora M., Li M., Maineult A., Tang Y.B. (2011) Pore connectivity, permeability and electrical formation factor: a new model and comparison to experimental data, J. Geophys. Res. 116, B11204.

Bernabé Y., Maineult A. (2015) Physics of porous media: fluid flow through porous media, Treatise on Geophysics, $2^{\text {nd }}$ ed., Vol. 11: Resources in the Near-Surface Earth, Schubert G. (ed.), pp. 19-41. 
Berryman J.G. (1992a) Effective stress for transport properties of inhomogeneous porous rock, J. Geophys. Res. - Sol. Earth 97, 17409-17424.

Berryman J.G. (1992b) Exact effective-stress rules in rock mechanics, Phys. Rev. A 46, 6, 3307-3311.

Berryman J.G. (1993) Effective-stress rules for pore-fluid transport in rocks containing two minerals, Int. J. Rock Mech. Min. Sci. \& Geomech. Abstr. 30, 1165-1168.

Biella G., Lozej A., Tabacco I. (1983) Experimental study of some hydrogeophysical properties of unconsolidated porous media, Ground Water 21, 741-751.

Biella G., Tabacco I. (1981) The influence of grain size on the relations between resistivity, porosity and permeability in unconsolidated formations, Boll. Geof. Teor. Appl. 23, 43-58.

Blair S.C., Berge P.A., Berryman J.G. (1996) Using two-point correlation functions to characterize microgeometry and estimate permeabilities of sandstones and porous glass, J. Geophys. Res. - Sol. Earth 101, 20359-20375.

Bourbié T., Zinszner B. (1985) Hydraulic and acoustic properties as a function of porosity in Fontainebleau sandstone, J. Geophys. Res. - Sol. Earth 90, 11524-11532.

Clennell M.B. (1997) Tortuosity: a guide through the maze, Geol. Soc. London, Spec. Pub. 122, 299-344.

Coelho D., Thovert J.-F., Adler P.M. (1997) Geometrical and transport properties of random packings of spheres and aspherical particles, Phys. Rev. E 55, 1959-1978.

Doyen P.M. (1988) Permeability, conductivity, and pore geometry of sandstone, J. Geophys. Res. - Sol. Earth 93, 7729-7740.

Fischmeister H.F., Arzt E., Olsson L.R. (1978) Particle deformation and sliding during compaction of spherical powders: a study by quantitative metallography, Powder Metallurgy 4, 179-187.

Fredrich J.T., Greaves K.H., Martin J.W. (1993) Pore geometry and transport properties of Fontainebleau sandstone, Int. J. Rock Mech. Min. Sci. \& Geomech. Abstr. 30, 691-697.

Glover P.W.J., Hole M.J., Pous J. (2000) A modified Archie's law for two conducting phases, Earth Planet. Sci. Lett. 180, 369-383.

Glover P.W.J., Zadjali I.I., Frew K.A. (2006) Permeability prediction from MICP and NMR data using an electrokinetic approach, Geophys. 71, F49-F60.

Glover P.W.J. (2009) What is the cementation exponent? A new interpretation, The Leading Edge 28, 82-85.

Glover P.W.J., Walker E. (2009) Grain-size to effective pore-size transformation derived from electrokinetic theory, Geophys. 74, E17-E29.

Glover P.W.J., Déry N. (2010) Streaming potential coupling coefficient of quartz glass bead packs: dependence on grain diameter, pore size, and pore throat radius, Geophys. 75, F225-F241.
Guyon E., Oger L., Plona T.J. (1987) Transport properties in sintered porous media composed of two particle sizes, J. Phys. D: Appl. Phys. 20, 1637-1644.

Li S.X., Pengra D.B., Wong P.-z. (1995) Onsager's reciprocal relation and the hydraulic permeability of porous media, Phys. Rev. E 51, 5748-5751.

Lindquist W.B., Venkatarangan A., Dunsmuir J., Wong T.-f. (2000) Pore and throat size distributions measured from synchrotron X-ray tomographic images of Fontainebleau sandstones, J. Geophys. Res. - Sol. Earth 105, 21509-21527.

Paterson M.S. (1983) The equivalent channel model for permeability and resistivity in fluid-saturated rocks - a reappraisal, Mech. Mater. 2, 345-352.

Petford N., Davidson G., Miller J.A. (2001) Investigation of the petrophysical properties of a porous sandstone sample using confocal scanning laser microscopy, Pet. Geosci. 7, 99-105.

Revil A., Cathles L.M. (1999) Permeability of shaly sands, Water Resour. Res. 35, 651-662.

Revil A., Glover P.W.J. (1997) Theory of ionic surface electrical conduction in porous media, Phys. Rev. B 55, 1757-1773.

Revil A., Glover P.W.J. (1998) Nature of surface electrical conductivity in natural sands, sandstones and clays, Geophys. Res. Lett. 25, 691-694.

Revil A., Kessouri P., Torres-Verdin C. (2014) Electrical conductivity, induced polarization, and permeability of the Fontainebleau sandstone, Geophys. 79, D301-D318.

Roberts J.N., Schwartz L.M. (1985) Grain consolidation and electrical conductivity in porous media, Phys. Rev. B 31, 5990-5997.

Schwartz L.M., Kimminau S. (1987) Analysis of electrical conduction in the grain consolidation model, Geophys. 52, 1402-1411.

Walsh J.B., Brace W.F. (1984) The effect of pressure on porosity and the transport properties of rock, J. Geophys. Res. - Sol. Earth 89, 9425-9431.

Wong P.-z., Koplik J., Tomanic J. (1984) Conductivity and permeability of rocks, Phys. Rev. B 30, 6606-6614.

Yale D.P. (1984) Network modeling of flow, storage and deformation in porous rocks, Ph.D. Thesis, Stanford University.

Zhu W., David C., Wong T.-f. (1995) Network modeling of permeability evolution during cementation and hot isostatic pressing, $J$. Geophys. Res. 100, 15451-15464.

Zhu W., Evans B., Bernabé Y. (1999) Densification and permeability reduction in hot-pressed calcite: a kinetic model, J. Geophys. Res. 104, 25501-25511.

Manuscript submitted in May 2015 Manuscript accepted in October 2015 Published online in June 2016

Cite this article as: Y. Bernabé, M. Li, Y.-B. Tang and B. Evans (2016). Pore Space Connectivity and the Transport Properties of Rocks, Oil Gas Sci. Technol 71, 50. 


\section{APPENDIX}

\section{Appendix A: Hydraulic Radius}

Unconsolidated packing of monodisperse spheres: We consider a volume $V_{0}$ containing $n$ spheres. The porosity and hydraulic radius are given by:

$$
\phi=\frac{V_{0}-n \frac{4 \pi R_{G}^{3}}{3}}{V_{0}}
$$

and

$$
r_{H}=2 \frac{V_{0}-n \frac{4 \pi R_{G}^{3}}{3}}{n 4 \pi R_{G}^{2}}
$$

We can calculate $V_{0}$ from Equation (A1) and plug it into (A2). Since $V_{0}$ is proportional to the number of spheres, $n$ cancels out and Equation (7) is obtained. The same derivation applies to sand grains, except that the surface of the grains must be written $n f_{G}$ $4 \pi R_{G}^{2}$, where $f_{G}$ is the asphericity factor.

Sintered glass beads: After sintering the glass beads volume $V_{0}$ is reduced to $V_{\text {snt }}$. Since the volume of solid does not change during sintering, the porosity of the sintered glass beads can be written as:

$$
\phi=\frac{V_{s n t}-n \frac{4 \pi R_{G}^{3}}{3}}{V_{s n t}}
$$

Similarly, the hydraulic radius is given by:

$$
r_{H}=2 \frac{n \frac{4 \pi R_{G}^{3}}{3} \frac{\phi}{1-\phi}}{n A_{G}}
$$

where $A_{G}$ is the average surface area of a single sintered sphere. A sintered sphere can approximately be represented as a truncated sphere with $z$ spherical caps removed (Roberts and Schwartz, 1985; Schwartz and Kimminau, 1987), where $z$ is the average number of grain contacts per sphere, which can be estimated as $z=6 \phi^{-1 / 5}$ based on the experimental data of Fischmeister et al. (1978) on plastically compressed packings of monodisperse bronze spheres. Because the grain contacts are not all formed at the same time during sintering, we must evaluate the number $d z$ of new grain contacts forming when the porosity decreases from $\phi^{*}$ to $\phi^{*}-d \phi^{*}$ (where * denotes a running porosity variable, between the initial and final values, $\phi_{0}$ and $\phi<\phi_{0}$, respectively) and the depth $h^{*}$ reached by the corresponding spherical caps at the end of the sintering process, i.e., when $\phi^{*}$ approaches $\phi$. By differentiation we obtain:

$$
d z=\frac{6}{5}\left(\phi^{*}\right)^{-\frac{6}{5}} d \phi^{*}
$$

The linear dimension of the volume of the sintered spheres must be proportional to the distance between contacting spheres, implying:

$$
\left(\frac{V}{V^{*}}\right)^{\frac{1}{3}}=\left(\frac{1-\phi^{*}}{1-\phi}\right)^{\frac{1}{3}}=\frac{R_{G}-h^{*}}{R_{G}}
$$

where only the new grain contacts formed at $\phi^{*}$ are considered. Remembering that the surface of a spherical cap of depth $h^{*}$ is $2 \pi R_{G} h^{*}$, we obtain $A_{G}$ equal to:

$$
A_{G}=4 \pi R_{G}^{2}-\int_{\phi<\phi_{0}}^{\phi_{0}} \frac{12 \pi R_{G}^{2}}{5}\left(1-\left(\frac{1-\phi^{*}}{1-\phi}\right)^{\frac{1}{3}}\right)\left(\phi^{*}\right)^{-\frac{6}{5}} d \phi^{*}
$$




\section{Appendix B: Hydraulic and Electrical Conductance of Constricted Pipes}

We consider a frustum-shaped pipe of length $l_{c}$. We call $r_{1}$ and $r_{2}>r_{1}$ the radii of the narrow and wide bases, respectively, and $x$ the coordinate along the pipe axis. We assume that fluid flow through this pipe is laminar and obeys Reynold's equations, i.e., the locally expressed Poiseuille law is valid for any value of $x$, a condition most likely met in long pipes. The flow rate $q$ is constant along the pipe and given by:

$$
q=\frac{\pi \frac{d p}{d x}(x) r(x)^{4}}{8 \eta}
$$

where $p(x)$ is the fluid pressure at $x, r(x)=r_{1}+x\left(r_{2}-r_{1}\right) / l_{c}$ the cross-sectional radius and $\eta$ the fluid viscosity. We can thus write:

$$
\frac{d}{d x}\left(r^{4} \frac{d p}{d x}\right)=0
$$

After double integration of Equation (B2), we find:

$$
q=\frac{3 \pi|\Delta p| r_{1}^{3} r_{2}^{3}}{8 \eta l_{c}\left(r_{1}^{2}+r_{1} r_{2}+r_{2}^{2}\right)}
$$

where $|\Delta p|$ is the absolute value of the apparent pressure difference between the pipe faces. A similar expression can be written for a straight, cylindrical pipe of radius $r=r_{H}$ and length $l$ :

$$
q=\frac{\pi|\Delta p| r_{H}^{4}}{8 \eta l}
$$

Thus, the ratio $g_{H}$ of the frustum pipe hydraulic conductance to that of a cylindrical pipe is:

$$
g_{H}=\frac{3 l r_{1}^{3} r_{2}^{3}}{l_{c}\left(r_{1}^{2}+r_{1} r_{2}+r_{2}^{2}\right) r_{H}^{4}}
$$

The electrical resistance $R_{c}$ of a frustum pipe saturated with a solution of resistivity $\rho_{s}$ is:

$$
R_{c}=\int_{0}^{l_{c}} \frac{\rho_{s} d x}{\pi r(x)^{2}}=\frac{l_{c} \rho_{s}}{\pi r_{1} r_{2}}
$$

Likewise, the resistance of a cylindrical pipe is equal to $l \rho_{s} /\left(\pi r_{H}^{2}\right)$ and the ratio $g_{E}$ of the electrical conductances is:

$$
g_{E}=\frac{l r_{1} r_{2}}{l_{c} r_{H}^{2}}
$$

